\title{
Structure and Morphology of ACEL ZnS:Cu,Cl Phosphor Powder Etched by Hydrochloric Acid
}

\author{
Robert Withnall, ${ }^{\mathrm{Z}}$ Jack Silver, Terry G. Ireland, \\ George R. Fern, and Paul J. Marsh
}

Wolfson Centre for Materials Processing, Brunel University, Uxbridge, Middlesex UB8 3PH, United Kingdom

\begin{abstract}
Despite many researches over the last half century, the mechanism of ac powder electroluminescence remains to be fully elucidated and, to this end, a better understanding of the relatively complex structure of alternate current electroluminescence (ACEL) phosphors is required. Consequently, the structure and morphology of $\mathrm{ZnS}: \mathrm{Cu}, \mathrm{Cl}$ phosphor powders have been investigated herein by means of scanning electron microscopy (SEM) on hydrochloric acid-etched samples and X-ray powder diffraction. The latter technique confirmed that, as a result of two-stage firing during their synthesis, the phosphors were converted from the high temperature hexagonal (wurtzite) structure to the low temperature cubic (sphalerite) polymorph having a high density of planar stacking faults. Optical microscopy revealed that the crystal habit of the phosphor had the appearance of the hexagonal polymorph, which can be explained by the sphalerite pseudomorphing of the earlier wurtzite after undergoing the hexagonal to cubic phase transformation during the synthesis. SEM micrographs of the hydrochloric-etched phosphor particles revealed etch pits, a high density of planar stacking faults along the cubic [111] axis, and the pyramids on the (111) face. These observations were consistent with unidirectional crystal growth originating from the face showing the pyramids. (C) 2009 The Electrochemical Society. [DOI: 10.1149/1.3207950] All rights reserved.
\end{abstract}

Manuscript submitted May 26, 2009; revised manuscript received July 29, 2009. Published September 8, 2009.

The pioneer of ac powder electroluminescence (EL) is Destriau, ${ }^{1}$ who reported light emission from a suspension of ZnS-based powder in castor oil when it was subjected to an ac electric field. Although the mechanism of ac powder EL has still not been fully elucidated, many investigations into the phenomenon have been carried out to advance our understanding. Fischer ${ }^{2,3}$ developed a model involving bipolar field emission into the zinc sulfide lattice from the opposite ends of copper sulfide needles embedded within it. According to this model, subsequent trapping of the injected holes at copper activator centers and charge recombination of the trapped holes with mobile electrons on field reversal gave rise to light emission in the form of lines or striations extending through most of the diameter of the particles.

Although the Fischer model is still widely accepted, there have been few reports on the direct observation of the copper sulfide phase, which is implicated in the field emission process in the model. One of these is a short article by Ono et al., ${ }^{4}$ reporting the detection of copper sulfide needles in the form of $\mathrm{Cu}_{2} \mathrm{~S}$ by means of $\mathrm{X}$-ray absorption near-edge structure spectroscopy; another is a short report by Guo et al., who observed a higher concentration of copper in the twin boundary areas and crystal streaks of sphalerite using high resolution electron microscopy and scanning electron microanalysis; and a third is a recent article by Warkentin et al., ${ }^{6}$ who maintained that copper sulfide is present in the form of tiny CuS-like clusters by means of extended X-ray absorption fine structure spectroscopy. However, others have found no evidence of copper sulfide needles, ${ }^{7,8}$ thus the precise nature of the role of copper sulfide clusters and the charge generation process presently remain open to conjecture.

A further question about the mechanism of ac powder EL concerns the structural requirements of the host zinc sulfide lattice. Zinc sulfide crystallizes in the form of either the sphalerite or wurtzite polymorphs, the former is cubic close-packed and shows the $\mathrm{AB}$ CABC... stacking pattern (3C modification) along the [111] directions, where $\mathrm{A}, \mathrm{B}$, and $\mathrm{C}$ represent $\mathrm{Zn}-\mathrm{S}$ double layers, whereas the latter is hexagonal close-packed and exhibits the ABABAB... stacking pattern ( $2 \mathrm{H}$ modification) along the $c$ axis. ${ }^{9}$ The $3 \mathrm{C}$ modification is the lower temperature form, with the $3 \mathrm{C} \rightarrow 2 \mathrm{H}$ phase transformation being observed to occur at a temperature of ca. $1024^{\circ} \mathrm{C}$. The $2 \mathrm{H}$ modification is metastable below $1024^{\circ} \mathrm{C}$ because the $2 \mathrm{H}$ crystal eventually transforms into a twinned $3 \mathrm{C}$ structure if it is annealed at

${ }^{\text {z } E-m a i l: ~ r o b e r t . w i t h n a l l @ b r u n e l . a c . u k ~}$ sufficiently high temperatures (below the phase transition temperature) for a long enough time. ${ }^{10}$ When a little CdS or MnS is admixed with the $\mathrm{ZnS}$, however, the solid solution has been reported to partially undergo a $2 \mathrm{H} \rightarrow 6 \mathrm{H}$ phase transformation on annealing instead. ${ }^{11-14}$ A third polymorph of $\mathrm{ZnS}$, matraite, was reported some time ago, ${ }^{15}$ but it is apparently not a distinct mineral species and can be treated the same as (111)-twinned sphalerite according to a recent report. ${ }^{16}$

In $\mathrm{ZnS}$, however, the stacking pattern can deviate from the above-mentioned ideal ABCABC... cubic close-packed and ABABAB ... hexagonal close-packed sequences, giving many different polytypes. Polytypism in mineral $\mathrm{ZnS}$ crystals was discovered by Frondel and Palache, ${ }^{17,18}$ and $\mathrm{ZnS}$ is one of the widely known polytypic materials; polytypism is defined as the building up of solids from identical modular layers that differ only in their stacking orientation, whereby one can assume a crystalline order in the twodimensional modular layers but not necessarily in the third dimension (the layer stacking direction). ${ }^{1,20}$ This is exemplified by $\mathrm{ZnS}$, for which over 185 polytypes have been reported to have different stacking sequences of $\mathrm{Zn}-\mathrm{S}$ layers. ${ }^{10,21,22}$ The polytypism of the host zinc sulfide lattice of a $\mathrm{ZnS}: \mathrm{Cu}, \mathrm{Cl}$ phosphor depends on the processing conditions of the phosphor, and it is clearly of interest to study how it affects the mechanism of ac powder EL.

\section{Experimental}

Synthesis.- Osram Sylvania noncoated and aluminum oxyhydroxide coated alternating current electroluminescence (ACEL) $\mathrm{ZnS}$ :Cu-based phosphors (types 728 and GG43, respectively), luminescent grade $\mathrm{ZnS}$ (Towanda), and Kerune noncoated ACEL $\mathrm{ZnS}: \mathrm{Cu}$-based phosphor (Changchun, China) were obtained from the manufacturers. Copper sulfate pentahydrate, sodium, magnesium and barium chlorides, potassium cyanide, zinc oxide (all from Sigma Aldrich, Gillingham, U.K.), and hydrochloric acid (Merck, Poole, U.K.) were used without further purification.

The commonly adopted synthetic procedure for the preparation of ACEL $\mathrm{ZnS}: \mathrm{Cu}, \mathrm{X}(\mathrm{X}=\mathrm{Cl}, \mathrm{Br})$ phosphor powders involves a twostage firing, the first stage involving annealing of a mixture of $\mathrm{ZnS}$, copper salts, and fluxes at a temperature range of $1100-1400^{\circ} \mathrm{C}$. This is followed by a mechanical treatment, such as ballmilling, before the second stage firing is carried out at a lower temperature range of $500-1000^{\circ} \mathrm{C}^{23}$ Based on this general procedure, many patents have been granted to different approaches to the synthesis of these phosphors as well as for ACEL device architecture. ${ }^{24}$ The synthesis of ACEL $\mathrm{ZnS}: \mathrm{Cu}$ powders in terms of materials is relatively inexpensive, but the production is costly and complex. 
A typical synthesis of in-house ACEL $\mathrm{ZnS}: \mathrm{Cu}, \mathrm{Cl}$ using the method reported by Takemura et al. ${ }^{25}$ is described below. Osram Sylvania $\mathrm{ZnS}(50 \mathrm{~g})$ was stirred with an aqueous solution $\left(50 \mathrm{~cm}^{3}\right)$ of copper sulfate pentahydrate $(0.15 \mathrm{~g})$ for $0.5 \mathrm{~h}$. The slurried material was dried overnight at $150^{\circ} \mathrm{C}$. The dry material $(25 \mathrm{~g})$ was thoroughly mixed with magnesium chloride $(0.5 \mathrm{~g})$, barium chloride $(2 \mathrm{~g})$, and sodium chloride $(2.5 \mathrm{~g})$. The mixed material was then fired at $1150^{\circ} \mathrm{C}$ for $4 \mathrm{~h}$.

The fired material was washed with hydrochloric acid, dried, and then pressed at a uniaxial pressure of 1.24 tons $/ \mathrm{cm}^{2}$ for $5 \mathrm{~min}$. $\mathrm{ZnO}$ (5\% by weight) was added, and then the mixture was fired at $750^{\circ} \mathrm{C}$ for $1.5 \mathrm{~h}$. The fired material was washed again in hydrochloric acid, and after the $\mathrm{pH}$ had been adjusted, with potassium cyanide $(\mathrm{KCN})$.

The acid etching of the ACEL $\mathrm{ZnS}: \mathrm{Cu}, \mathrm{Cl}$ particles was accomplished using 6 and $12 \mathrm{M}$ hydrochloric acid for soft and hard etchings, respectively. The $\mathrm{ZnS}: \mathrm{Cu}, \mathrm{Cl}(1.0 \mathrm{~g})$ particles were placed in a centrifuge tube $\left(50 \mathrm{~cm}^{3}\right)$ along with hydrochloric acid $\left(10 \mathrm{~cm}^{3}\right)$ for soft and hard etchings, and the centrifuge tube was then placed on a set of motorized rollers and was gently rotated for the period of etching. After etching, the acid was decanted from the particles, washed with deionized water three times, filtered at the pump, and then dried in an oven at $150^{\circ} \mathrm{C}$ for $1 \mathrm{~h}$.

Instrumentation.- Field-emission scanning electron microscopy (FESEM) was used to study the particle sizes and morphologies of the samples using a Zeiss Supra 35 VP instrument (Oberkochen, Germany). The samples were mounted on carbon tabs attached to aluminum stubs.

All samples were dispersed in castor oil and placed in a standard Destriau cell comprising an aluminum electrode and an indium tin oxide-coated glass electrode for ACEL light emission measurements. ${ }^{1}$ A paste consisting of $1.4 \mathrm{~g}$ of phosphor and $1.0 \mathrm{~cm}^{3}$ of castor oil was prepared for each commercial phosphor sample, and $0.2 \mathrm{~g}$ of the paste was then transferred to a Destriau cell to ensure that the density of the phosphor material was the same in each case. The Destriau cell was excited with an alternating voltage of $110 \mathrm{~V}$ [root-mean-square (rms)] and a sine wave frequency of 400 $\mathrm{Hz}$ using a Newtons4th SLM2200 frequency generator and an LPA400 laboratory power amplifier (Newtons4th Ltd., Loughborough, U.K.)

The EL emission spectra and luminance measurements were obtained by means of a Jeti spectroradiometer (Specbos 1201, Jeti Technische Instrumente GmbH, Jena, Germany).

$\mathrm{X}$-ray powder diffraction measurements were made using a Bruker D8 powder diffractometer fitted with a nickel-filtered copper source and a LynxEye silicon strip detector. Data were recorded from 5 to $100^{\circ} 2 \theta$ at $293 \mathrm{~K}$, and the data were fitted using a standard refinement method by means of a Bruker AXS TOPAS version 3 Rietveld refinement computer software. The background was refined using a Chebychev polynomial and a correction for $2 \theta$ was made using a National Institute of Standards and Technology standard calibrant. For the crystallographic phases, the lattice parameters and the crystallite sizes were varied in the data fits, but the site occupancies, atomic coordinates, and thermal parameters were fixed.

\section{Results and Discussion}

The structures and morphologies of both hydrochloric acidetched commercial ZnS:Cu-based ACEL phosphor particles and hydrochloric acid-etched in-house synthesized $\mathrm{ZnS}: \mathrm{Cu}, \mathrm{Cl}$ ACEL particles are discussed below.

A typical powder X-ray diffraction (XRD) diffractogram obtained from samples of $\mathrm{ZnS}$ doped with $\mathrm{Cu}$ after firing at $1150^{\circ} \mathrm{C}$ for $4 \mathrm{~h}$, according to the procedure mentioned in the Experimental section, is shown in Fig. 1 (solid black trace). This has been fitted to reference diffractograms from the Cambridge Crystallographic Data Centre (CCDC) database ${ }^{26}$ (dotted black trace in Fig. 1). As expected, the diffractogram of the sample exhibits reflections that are characteristics of the $2 \mathrm{H}$ form of wurtzite (space group $P 63 m c$ ), as can be seen from the fit to the reference diffractogram of wurtzite

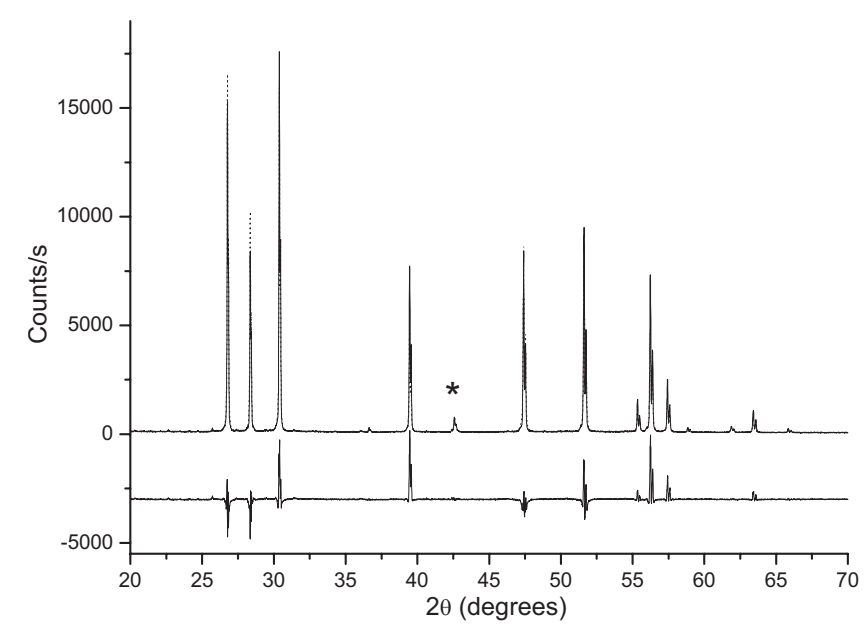

Figure 1. Overlay of powder XRD diffractogram of $\mathrm{ZnS}: \mathrm{Cu}, \mathrm{Cl}$ after firing at $1150^{\circ} \mathrm{C}$ for $4 \mathrm{~h}$ (solid black trace) and fit of wurtzite $2 \mathrm{H}$ structure, as explained in the text (dotted black trace). The asterisk indicates a peak due to a minor magnesium zinc oxide phase. The residual, after subtraction of the fit from the observed diffractogram, is shown by the solid black trace at the bottom.

2H (ICDD ref. no. 361450) in Fig. 1 (dotted black trace). A peak at a $2 \theta$ value of $42.5^{\circ}$ (denoted by an asterisk in Fig. 1) can be assigned to the (200) reflection from a small amount (ca. 5\%) of a magnesium zinc oxide phase (space group $F m \overline{3} m$ ) having the stoichiometry $\mathrm{Mg}_{0.75} \mathrm{Zn}_{0.25} \mathrm{O}$ by comparison with the reference diffractogram of this material (ICSD ref. no. 77066). This phase presumably originates from a reaction of the magnesium chloride that was used for the flux.

A powder XRD diffractogram, obtained from the commercial Osram Sylvania-uncoated ACEL phosphor (type 728), is shown by the solid black trace in Fig. 2, along with a fit of reference XRD diffractograms of sphalerite (ICDD ref. no. 50566), wurtzite $2 \mathrm{H}$ (ICDD ref. no. 361450), and wurtzite 6H (ICSD ref. no. 43392) from the CCDC database ${ }^{26}$ (dotted black trace in Fig. 2). The diffractogram of the sample shows the characteristic peaks of wurtzite $2 \mathrm{H}$, indicating that a percentage (ca. $24 \%$ ) of the high temperature hexagonal phase produced in the first stage firing persists after the mechanical treatment and the lower temperature second stage firing.

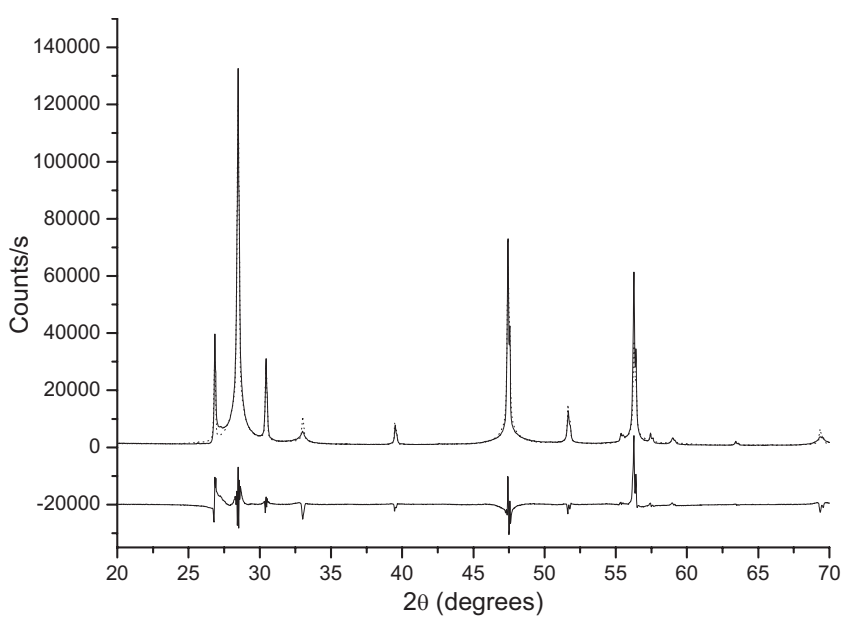

Figure 2. Overlay of powder XRD diffractogram of commercial Osram Sylvania type $728 \mathrm{ZnS}$ :Cu-based ACEL phosphor (solid black trace) and fit of reference sphalerite, wurtzite $2 \mathrm{H}$, and wurtzite $6 \mathrm{H}$ structures, as explained in the text (dotted black trace). The residual, after subtraction of the fit from the observed diffractogram, is shown by the solid black trace at the bottom. 


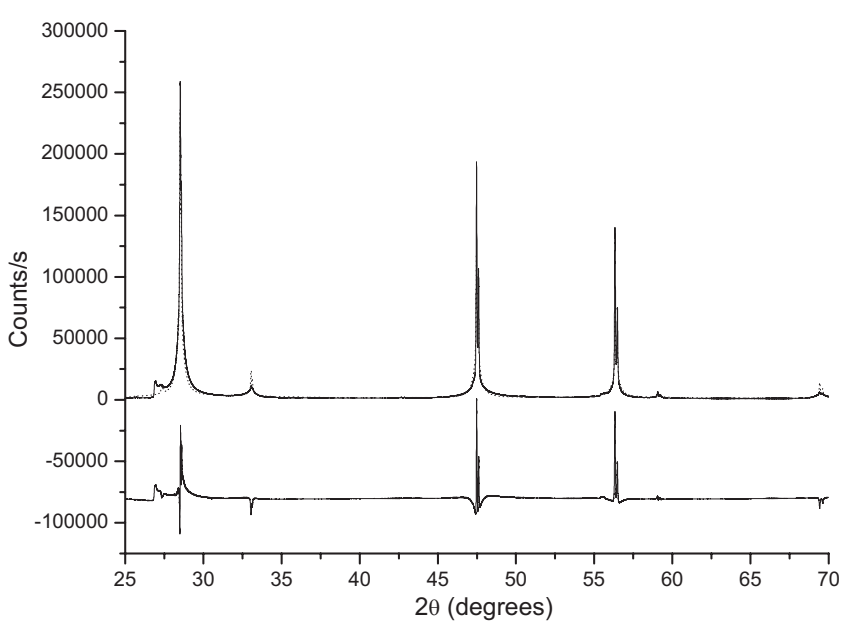

Figure 3. Overlay of powder XRD diffractogram of commercial Osram Sylvania type GG43 ZnS:Cu-based ACEL phosphor (solid black trace) and fit of reference sphalerite and wurtzite $6 \mathrm{H}$ structures, as explained in the text (dotted black trace). The residual, after subtraction of the fit from the observed diffractogram, is shown by the solid black trace at the bottom.

The predominant phase of the uncoated ACEL phosphor (type 728) is sphalerite (space group $F 43 \mathrm{~m}$ ), however, as is made evident by strong peaks at $2 \theta$ values of $28.5,47.5$, and $56.3^{\circ}$, which can be ascribed to the (111), (220), and (311) reflections, respectively. To improve the fit of the line shapes in Fig. 2, three reference XRD diffractograms of sphalerite were used, having different crystallite sizes of 18, 76, and $257 \mathrm{~nm}$. However, there is a small amount (ca. $6 \%$ ) of the $6 \mathrm{H}$ form of wurtzite (space group $P 63 \mathrm{mc}$ ), as can be seen by reflections at $2 \theta$ values of 27.4 and $28.6^{\circ}$, which can be ascribed to the (101) and (006) reflections, respectively.

The above-mentioned XRD data are consistent with two structural conversions taking place after the mechanical treatment and the second firing of the $\mathrm{ZnS}$ :Cu-based phosphor material. The major one is the $2 \mathrm{H}-3 \mathrm{C}$ conversion and the minor one is a $2 \mathrm{H}-6 \mathrm{H}$ transformation. The latter transformation can occur when small amounts of impurity, e.g., CdS and MnS, are present, ${ }^{11-14}$ and it has also been reported to occur in copper-doped zinc sulfide. ${ }^{27}$ Furthermore, transmission electron microscopy (TEM) studies of the commercial Osram Sylvania phosphor (type 728) report that the zinc sulfide lattice has a densely twinned sphalerite structure.

A powder XRD diffractogram, obtained from the commercial Osram Sylvania type GG43 ACEL phosphor, is shown by the solid black trace in Fig. 3, along with a fit (dotted black trace in Fig. 3) of reference XRD diffractograms of (111)-twinned sphalerite (matraite) ${ }^{16}$ as the major component (ca. 99.3\%) and a small amount (ca. $0.7 \%$ ) of wurtzite $6 \mathrm{H}$ (ICDD ref. no. 43392 ) ${ }^{26}$ Furthermore, to improve the fit of the line shapes in Fig. 3, two reference XRD diffractograms of (111)-twinned sphalerite were used, one having a crystallite size of $18 \mathrm{~nm}$ and the other with a crystallite size of 352 $\mathrm{nm}$. The absence of wurtzite $2 \mathrm{H}$ in this phosphor is due to its complete conversion to (111)-twinned sphalerite, along with a small amount (ca. $0.7 \%$ ) of wurtzite $6 \mathrm{H}$ (indicated by reflections at $2 \theta$ values of 27.4 and $28.6^{\circ}$ ) as a result of the mechanical treatment and the second stage firing (see Experimental procedure above). Furthermore, the absence of wurtzite $2 \mathrm{H}$ and the predominance of (111)twinned sphalerite in this phosphor strongly suggest that it is the latter phase and not the former one that is responsible for ACEL.

For the in-house $\mathrm{ZnS}: \mathrm{Cu}, \mathrm{Cl}$ phosphors synthesized by the Takemura method, ${ }^{25}$ it can be seen from Fig. 4 that the $2 \mathrm{H}-3 \mathrm{C}$ and $2 \mathrm{H}-6 \mathrm{H}$ transformations take place as a result of the uniaxial pressure applied to the sample following the first firing, according to the procedure described in the Experimental section. Figure 4 compares the powder XRD diffractograms of $\mathrm{ZnS}: \mathrm{Cu}, \mathrm{Cl}$ before (solid black trace) and after (dotted black trace) applying the uniaxial pressure.

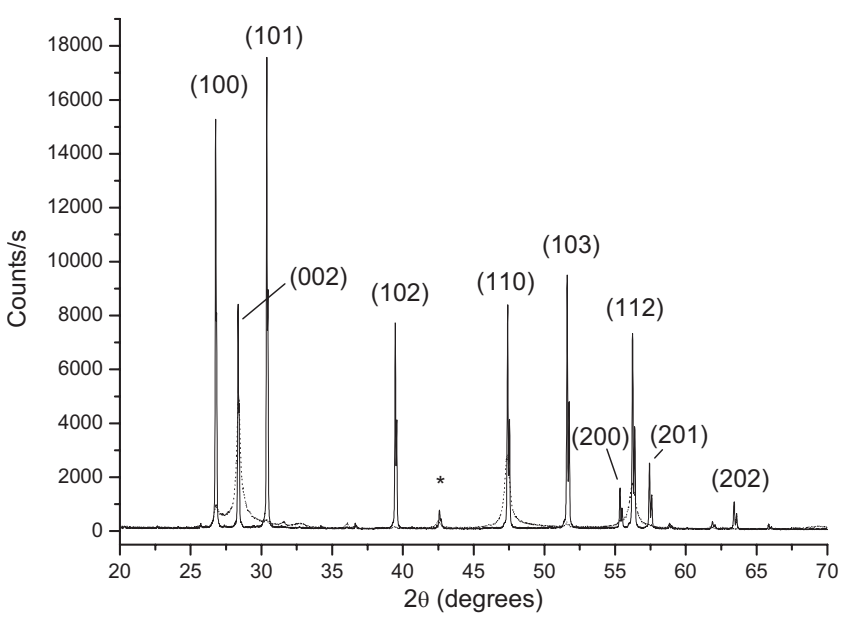

Figure 4. Powder XRD diffractograms of $\mathrm{ZnS}: \mathrm{Cu}, \mathrm{Cl}$ after firing at $1150^{\circ} \mathrm{C}$ for $4 \mathrm{~h}$ (solid black trace), and the same sample after uniaxial pressure treatment (dotted black trace). The asterisk indicates a peak due to a minor magnesium zinc oxide phase.

The former diffractogram was previously shown in Fig. 1 and is due to wurtzite $2 \mathrm{H}$ and a small amount of magnesium zinc oxide (as previously explained). The dotted black trace in Fig. 4 exhibits broad reflections at $2 \theta$ values of $28.4,47.4$, and $56.2^{\circ}$ that are ascribed to the (002), (110), and (112) reflections (equivalent hexagonal indexes) of sphalerite, respectively; these correspond to the above-mentioned sharper reflections for twinned sphalerite in Fig. 2 and 3. Similarly, a weak, broad reflection centered at a $2 \theta$ value of ca. $26.8^{\circ}$ in Fig. 4 can be ascribed to disordered wurtzite $6 \mathrm{H}$, which was also identified in Fig. 2 and 3. The $h, k$, and $l$ indexes of the wurtzite $2 \mathrm{H}$ reflections are shown in Fig. 4 , from which it can be seen that the conversion of wurtzite $2 \mathrm{H}$ to twinned sphalerite involves a loss in intensity of wurtzite $2 \mathrm{H}$ Bragg reflections, for which $h-k \neq 0(\bmod 3)$, as predicted by a theory of transformations involving nonrandom nucleation of stacking faults in the $2 \mathrm{H}$ structure. ${ }^{28}$

The observation reported herein that the wurtzite $2 \mathrm{H}$ to twinned sphalerite and the $2 \mathrm{H}-6 \mathrm{H}$ transformations are induced by uniaxial pressure is consistent with the observations of Shackar et al. ${ }^{29}$ They studied the effect of plastic deformation on single crystals of wurtzite $2 \mathrm{H}$ by applying mechanical stress at room temperature with the aid of a knife edge or a needle and reported that this induced the transformation of wurtzite $2 \mathrm{H}$ to disordered $3 \mathrm{C}$ and $6 \mathrm{H}$ structures. It is therefore apparent that extended stresses, such as those induced by the application of uniaxial pressure, can cause wurtzite $2 \mathrm{H}$ to undergo this transformation by nucleating stacking faults. In addition, the transformation apparently involves deformation faults rather than layer displacement faults because the 10.0 and 10.1 reflections of wurtzite $2 \mathrm{H}$ crystals are equally broadened early on in the transformation. ${ }^{28}$

A mild etching with hydrochloric acid was found to have a beneficial influence on the luminance of all the ACEL phosphor samples. Typical sets of results are given in Tables I and II for Osram Sylvania type 728 and Kerune noncoated $\mathrm{ZnS}: \mathrm{Cu}$-based phosphors, respectively. After etching the Osram Sylvania type 728 phosphor for $2 \mathrm{~min}$, the ACEL luminance increases by around $14 \%$ (see Table I), which is a significant improvement. This improvement in luminance is most likely due to an increased optical outcoupling due to the greater roughness of the particles. Etching for longer periods of time resulted in a decrease in ACEL luminance as the surface material was progressively stripped away, with the ACEL luminance dropping to $76 \%$ of its original value after etching for 60 min. The dominant emission wavelength was monitored for both ACEL (110 V rms and $400 \mathrm{~Hz}$ ) and photoluminescence (PL) (under $366 \mathrm{~nm}$ excitation). For ACEL, the dominant wavelength shifted 
Table I. ACEL (110 V rms, $400 \mathrm{~Hz}$ ) and PL (under $366 \mathrm{~nm}$ excitation) results for uncoated Osram Sylvania ACEL ZnS:Cu-based phosphor particles (type 728) after etching with hydrochloric acid for different time periods over the 0-60 min range.

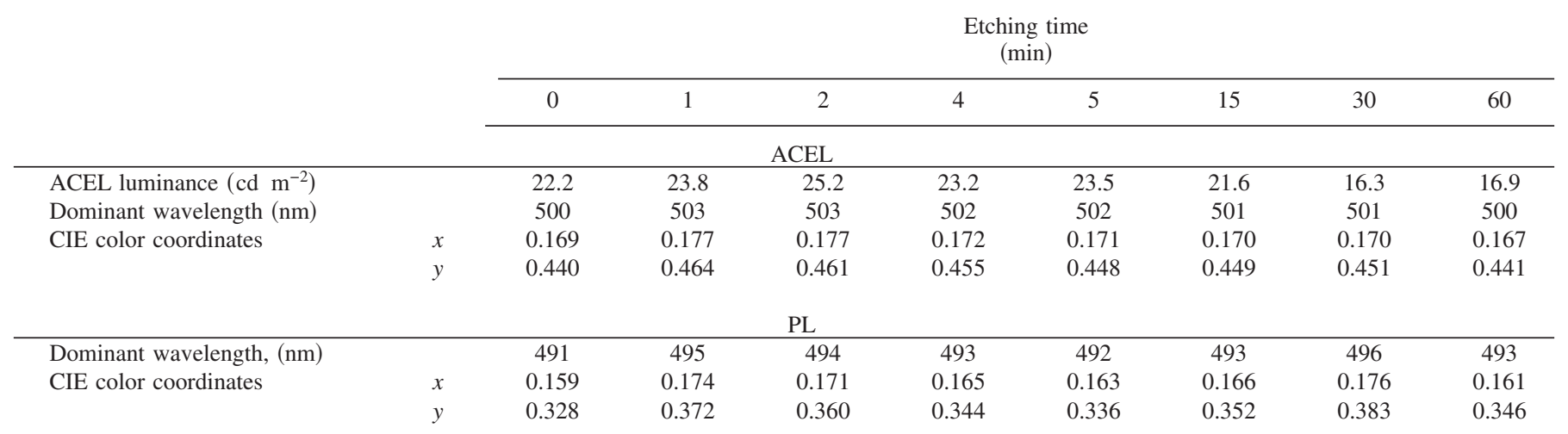

from 500 to $503 \mathrm{~nm}$ when the luminance reached a maximum after a 2 min etching time, then the dominant wavelength went back to $500 \mathrm{~nm}$ progressively, and the ACEL luminance decreased, following longer etching times. This small wavelength redshift was reproducible and could be due to the nonuniformity of the copper concentration that results from the cyanide washing procedure, which removes copper from the surfaces of the ACEL phosphor particles. For photoluminescence (PL), the dominant wavelength fluctuated between 491 and $496 \mathrm{~nm}$ without any discernible pattern. The CIE color chromaticity coordinates of both the ACEL and PL emissions showed slight changes consistent with the movement of the dominant wavelengths. Similar trends in the ACEL and PL properties were observed when the Kerune noncoated phosphor was etched with hydrochloric acid (see Table II).

Figure 5 shows scanning electron microscopy (SEM) micrographs of hydrochloric acid-etched commercial Osram Sylvania ACEL ZnS:Cu-based phosphor (type 728) particles that have undergone progressively longer periods of hydrochloric acid etching. The smooth surface of the particles that is seen before acid etching (see Fig. 5a) develops holes or "etch pits" after etching for 2 min (see Fig. 5b) and this coincides with the maximum ACEL luminance (see Table I). The surface has been stripped away after etching for 4 min, revealing the internal structure (see Fig. 5c). This structure exhibits pyramids at one end of the particle while the particle tapers down to a flat, featureless face at the opposite end. Stacks of well-defined layers are sandwiched between the two ends of the particle, and these are manifested by parallel striations or stripes on the surfaces of the particle, perpendicular to the layer stacking direction. These features are even more pronounced after etching the commercial Osram Sylvania ACEL ZnS:Cu-based phosphor (type 728) with hydrochloric acid for $15 \mathrm{~min}$ (see Fig. 5d).
A magnified FESEM image (see Fig. 6) of a single particle of the etched sample of Fig. 5c shows the anisotropy in more detail as well as the stacks of well-defined layers and pyramids at one end. The structural state of this sample is therefore that of a sphalerite showing a stacking sequence along one preferred direction, similar to that which has been reported by Fleet, ${ }^{22}$ for a natural $\mathrm{ZnS}$ mineral originating from Thomaston Dam, Connecticut. Taking into consideration the hexagonal habit of the grains, Fleet concluded that the Thomaston Dam sphalerite was pseudomorphing an earlier wurtzite from which it had transformed in the solid state. This explanation for the structure of the natural $\mathrm{ZnS}$ mineral from Thomaston Dam is entirely consistent with that of the synthetic $\mathrm{ZnS}$-based sample from Osram Sylvania (type 728), which has also undergone a solid-state transformation from wurtzite during the above-mentioned synthetic procedure. Furthermore, there appears to be a particularly striking similarity between the natural and synthetic samples. Fleet reported from his XRD investigation of the Thomaston Dam mineral that it had a twinned sphalerite structure containing admixed $6 \mathrm{H}$ wurtzite. ${ }^{22}$ Moreover, it has been suggested that the twinned microstructure of $\mathrm{ZnS}: \mathrm{Cu}$-based phosphors, such as that mentioned above for Osram Sylvania (type 728), may be significant to ACEL. ${ }^{30}$

The conversion from the hexagonal phase to the cubic one $(2 \mathrm{H}-$ $3 \mathrm{C})$ involves the rearrangement of the modular layer stacking pattern from $\mathrm{ABABAB}$... to $\mathrm{ABCABC}$..., and this can be achieved by the insertion of deformation faults into the structure. ${ }^{22,31}$ Layer stacking faults may be located randomly, nonrandomly, or periodically in the lattice. ${ }^{28}$ For the $2 \mathrm{H}-3 \mathrm{C}$ phase transformation, the faults are nonrandom because there is a higher probability of a fault occurring at every second layer (see Fig. 7a). The $2 \mathrm{H}-3 \mathrm{C}$ conversion, which occurs upon annealing the wurtzite modification at a tempera-

Table II. ACEL (110 V rms, $400 \mathrm{~Hz}$ ) and PL (under $366 \mathrm{~nm}$ excitation) results for uncoated Kerune ACEL ZnS:Cu-based phosphor particles

after etching with hydrochloric acid for different time periods over the 0-60 min range.

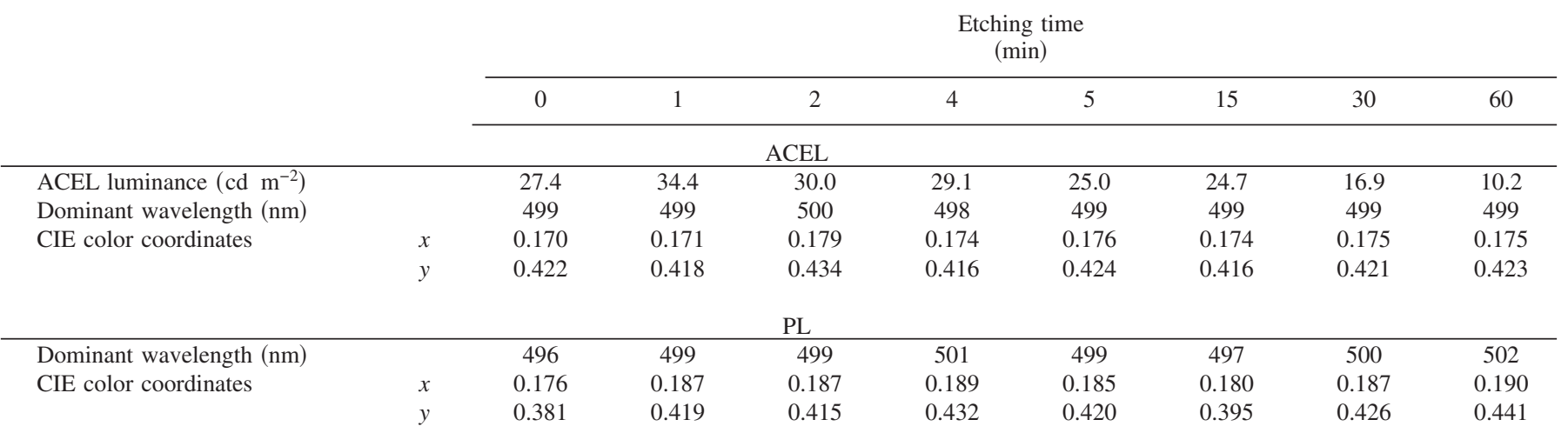



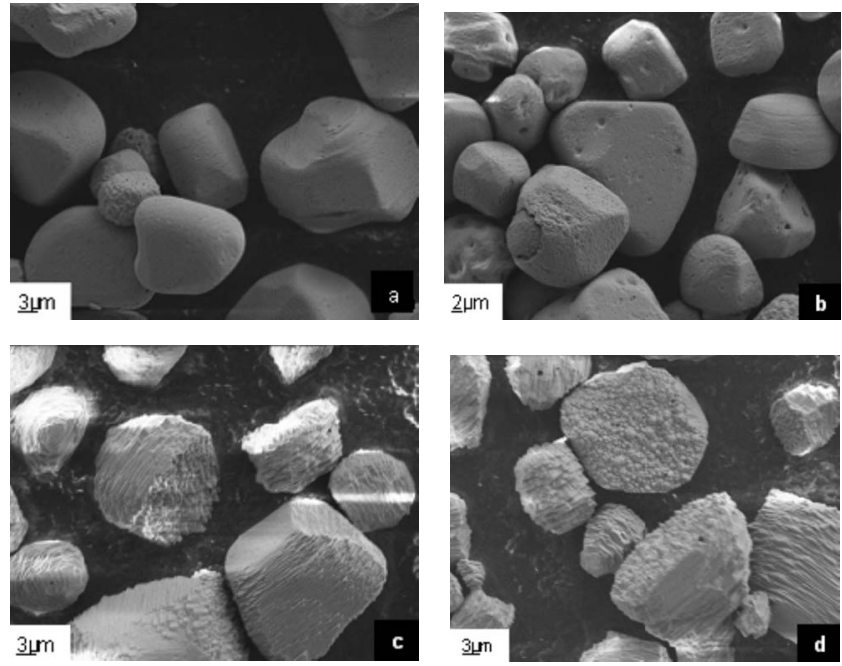

Figure 5. SEM micrographs of Osram Sylvania (type 728)-uncoated ACEL $\mathrm{ZnS}: \mathrm{Cu}$-based particles after varying periods of hydrochloric acid etching: (a) 1 , (b) 2, (c) 4, and (d) 15 min.

ture below the phase transition temperature $\left(1024^{\circ} \mathrm{C}\right)$, has been reported to be initiated by random insertion of deformation faults in the $2 \mathrm{H}$ structure ${ }^{28}$ these faults arise due to slippage of two parts of the crystal past each other. These provide nuclei for cubic regions to grow, following the nonrandom insertion of deformation faults at two layer separations (see Fig. 7a). The cubic structure produced is densely twinned along the [111] axis, and it should have statistically equal amounts of two twin orientations. Not only has such twinning been reported to occur in synthetic $\mathrm{ZnS}: \mathrm{Cu}$-based ACEL phosphors, ${ }^{7}$ it has also been observed in the Thomaston Dam sphalerite mineral, ${ }^{22}$ as mentioned above, and in the Fe-rich sphalerite minerals from the Trepča mines in Kosovo in which copper apparently concentrates at the $\{111\}$ twin boundaries. ${ }^{32}$ Another TEM study of a $\mathrm{ZnS}: \mathrm{Cu}$-based phosphor also indicated that copper may be associated with $\{111\}$ twin boundaries.

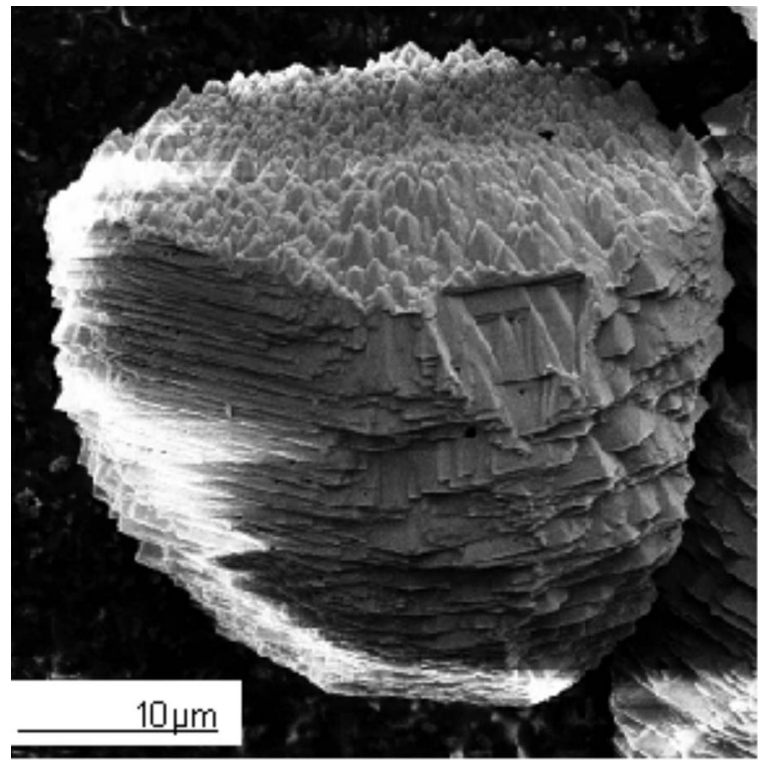

Figure 6. FESEM image of a single Osram Sylvania (type 728)-uncoated ACEL ZnS:Cu-based particle after etching with hydrochloric acid for 15 min, showing the layered structure along the [111] direction and pyramids at one end.

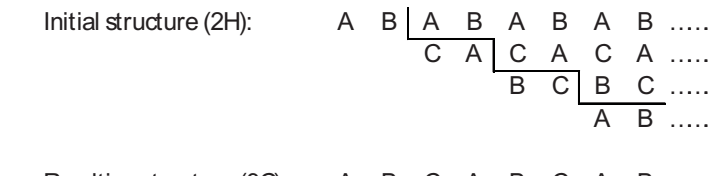

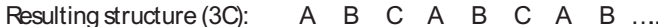

(a)

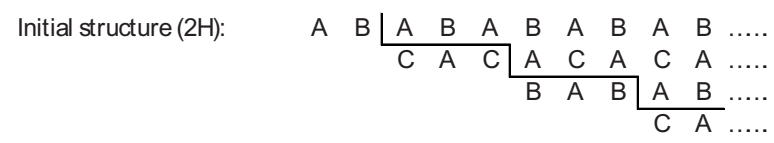

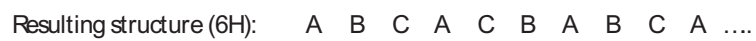

(b)

Figure 7. Schematic representations of nonrandom insertion of deformation faults at: (a) two layer separations in the $2 \mathrm{H}-3 \mathrm{C}$ transformation and (b) three layer separations in the $2 \mathrm{H}-6 \mathrm{H}$ transformation (taken from Ref. 11).

The $2 \mathrm{H}-6 \mathrm{H}$ transformation can arise as a result of the nonrandom insertion of deformation faults at three layer separations (see Fig. 7b). Single crystal XRD studies have indicated that the $2 \mathrm{H}-3 \mathrm{C}$ and $2 \mathrm{H}-6 \mathrm{H}$ transformations take place simultaneously in different regions of the same crystal. ${ }^{11}$

The (111)-twinned cubic structure as well as the $6 \mathrm{H}$ minor phase locks into place through this nonrandom faulting mechanism. This creates voids in the structure which are revealed by hydrochloric acid etching. The etched features are referred to as etch pits, and they are created at areas where the dislocations meet the surface, as can be seen in the SEM image in Fig. 5b. The etch pits are triangular and have been reported to appear on the $(\overline{1} \overline{1} 2)$ and $(11 \overline{2})$ faces of the sphalerite structure, having bases parallel to [1 $\overline{1} 0]$ and apices always pointing in the [111] direction, i.e., toward the (111) face ( $\mathrm{Zn}$ face), which exhibits the pyramids. ${ }^{33}$ In other words, the apices of the triangular etch pits point in the opposite direction to the [1 $\overline{1} \overline{1}]$ growth direction of the crystal, i.e., away from the ( $\overline{1} \overline{1} \overline{1})$ face $(\mathrm{S}$ face) ${ }^{33}$ Because the direction in which the apices of the triangular etch pits point is determined by the polar etching behavior, it has been previously concluded that the crystallographic polarity remains unchanged throughout the whole particle and is independent of changes in the crystal structure and disorder. ${ }^{34}$ Consequently, the triangular etch pits are identified with points of emergence of electrically charged dislocations, and they may be necessary but not sufficient for ACEL to be produced. ${ }^{35}$

The SEM images of commercial Osram Sylvania (type 728) ZnS:Cu-based ACEL phosphor particles that have been etched with $6 \mathrm{M}$ hydrochloric acid for periods of 0.5 and $1.0 \mathrm{~h}$ are shown in Fig. $8 \mathrm{a}$ and $\mathrm{b}$, respectively. These particles have diameters around twothirds of their original ones, but their ACEL luminance is still ca. $60 \%$ that of the particles before they were etched. This suggests that each exposed surface supports ACEL as a particle is progressively etched. This is consistent with ACEL light emission that is due to electron-hole recombination at the surface of the phosphor
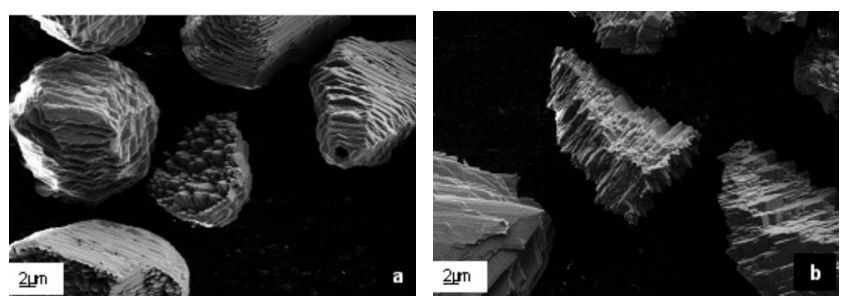

Figure 8. FESEM images of Osram Sylvania-uncoated ACEL ZnS:Cu-based particles that have been etched in hydrochloric acid for (a) 0.5 and (b) $1.0 \mathrm{~h}$. 

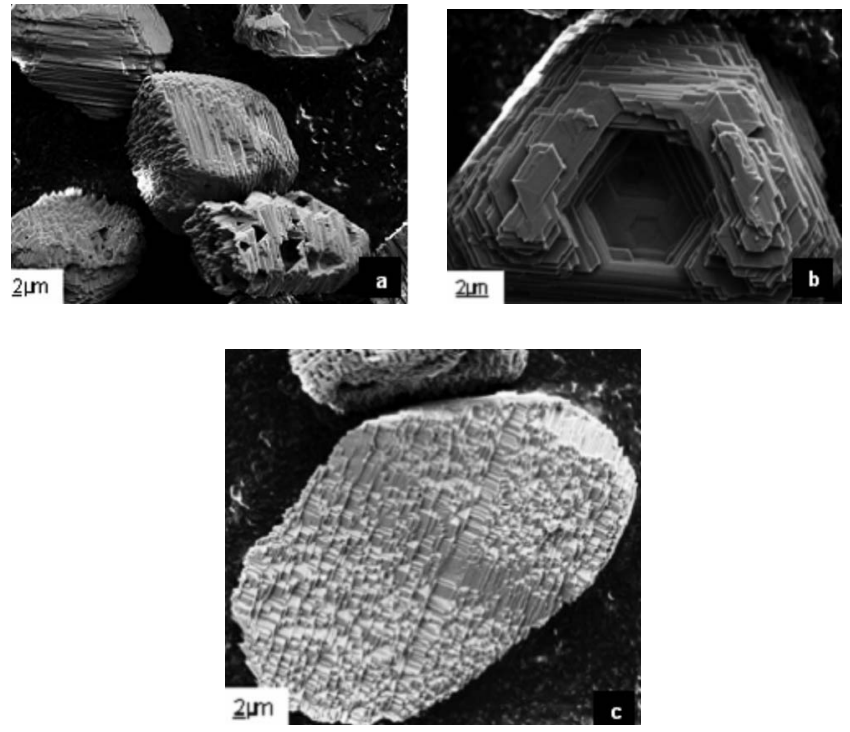

Figure 9. FESEM images of commercial Kerune ACEL ZnS:Cu-based particles that have been etched with $6 \mathrm{M}$ hydrochloric acid for $10 \mathrm{~min}$. (a) Particles displaying large triangular voids on the sides of the particles, (b) a view from the base of a particle, and (c) a cross section through a fractured particle.

particles, ${ }^{8}$ but it seems to indicate that charge recombination, giving rise to light emission, can also occur at surfaces that are exposed by acid etching. Some morphological changes occur after etching for longer periods of time, as can be seen by comparing Fig. 8a and b with Fig. 5c and d. First, the fine steps at the edges of the layers that stack in the [111] direction of this multiple-twinned sphalerite are more distinct after etching for a longer period (see Fig. 8a and b).

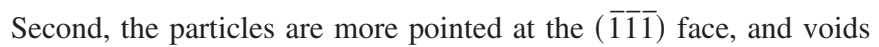
appear in some particles at their bases. However, the pyramids that occur on the (111) face persist throughout the etching and remain fairly constant in size.

The FESEM micrographs of commercial Kerune ACEL ZnS:Cubased particles that have been etched with $6 \mathrm{M}$ hydrochloric acid for 10 min are shown in Fig. 9. These particles, like the etched Osram Sylvania ACEL particles shown in Fig. 5d, exhibit pyramids at one end, which is presumably the (111) face of the multiple-twinned sphalerite structure. In Fig. 9b, the flat base of an etched particle has been etched to reveal a hexagonal hollow, and in Fig. 9c, a fractured cross section of one of the particles reveals stacked layers of pyramids, which indicate the growth axis of the particle.

The FESEM micrographs of in-house synthesized ACEL Zn$\mathrm{S}: \mathrm{Cu}, \mathrm{Cl}$ particles are shown in Fig. 10a and b. Again, voids are seen in particles (see Fig. 10a) and pyramids are seen on the (111) face of the particles, which have a twinned sphalerite structure (see Fig. $10 \mathrm{~b})$. These pyramids are smaller than those on the etched particles of the commercial samples most likely due to the differences in synthetic procedures, as the method reported by Takemura et al. ${ }^{25}$ was used to synthesize the in-house particles shown in Fig. 10a and b.

\section{Conclusions}

It has been shown herein that mild hydrochloric acid etching improves the luminance of ACEL $\mathrm{ZnS}$ :Cu-based particles. In addition, there remains a significant ACEL luminance from the exposed phosphor material that was previously in the interior of the particle before a large part of its volume was removed by hydrochloric acid etching.

A $2 \mathrm{H}-3 \mathrm{C}$ phase conversion results from mechanical treatment and annealing (in the $700-1000^{\circ} \mathrm{C}$ temperature range) of the wurtzite $2 \mathrm{H}$ form of $\mathrm{ZnS}: \mathrm{Cu}$-based phosphor precursors, which gives rise
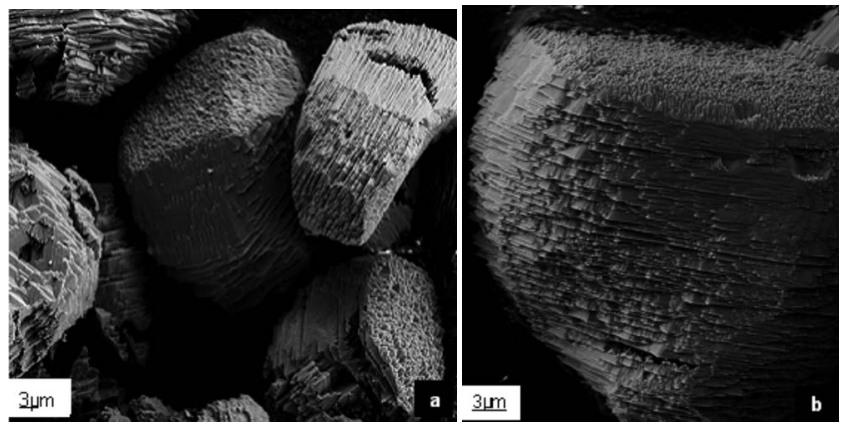

Figure 10. FESEM images of etched in-house ACEL $\mathrm{ZnS}: \mathrm{Cu}, \mathrm{Cl}$ particles synthesized using the method reported by Takemura et al. ${ }^{25}$ (a) Particles showing large voids and (b) a single etched particle showing a layered structure and small pyramids at one end.

to a (111)-twinned sphalerite structure containing nonrandom faults. The $2 \mathrm{H}-6 \mathrm{H}$ conversion also occurred under these conditions as a very minor pathway, possibly due to the effect of copper doping. ACEL was observed from $\mathrm{ZnS}$ :Cu-based phosphors, which had been transformed either partially or completely from the wurtzite $2 \mathrm{H}$ phase. Thus, the (111)-twinned sphalerite phase of the $\mathrm{ZnS}: \mathrm{Cu}-$ based phosphor appears to be implicated in the ac electroluminescent activity, as has previously been suggested. ${ }^{30}$ Pyramids are observed on the (111) faces of etched particles using SEM while at the opposite end of the particles, the ( $\overline{1} \overline{1} \overline{1})$ face appears smooth and flat. These observations are consistent with unidirectional crystal growth along the $[\overline{1} \overline{1}]$ direction. Triangular etch pits have been observed by means of SEM, which are identified with the dislocations present in the (111)-twinned sphalerite structure. These etch pits are believed to be necessary but not sufficient for ACEL. ${ }^{35}$ Finally, optical microscopy revealed that the crystal habit of the phosphor had the appearance of the hexagonal polymorph, which can be explained by the sphalerite pseudomorphing of the earlier wurtzite after undergoing the hexagonal to cubic phase transformation during the synthesis.

\section{Acknowledgments}

We are grateful to the EPSRC and DTI for supporting the ACELOOP and FLCOLOR programs, respectively. We are also grateful to the Technology Strategy Board-led Technology Program for funding the PLACES, SHAPEL, and ACTIVEL programs.

Brunel University assisted in meeting the publication costs of this article.

\section{References}

1. G. Destriau, J. Chem. Phys., 33, 587 (1936).

2. A. G. Fischer, J. Electrochem. Soc., 109, 1043 (1962).

3. A. G. Fischer, J. Electrochem. Soc., 110, 733 (1963).

4. Y. Ono, N. Shiraga, H. Kadokura, and K. Yamada, Inst. Electron Inform. Commun Eng. Tech. Rep., 89, 378 (1990).

5. C.-X. Guo, S. Y. Zhang, B.-L. Li, and L. Xu, J. Lumin., 40-41, 782 (1988)

6. M. Warkentin, F. Bridges, S. A. Carter, and M. Anderson, Phys. Rev. B, 75, 075301 (2007).

7. A. C. Wright and I. V. F. Viney, Philos. Mag. B, 81, 279 (2001)

8. N. E. Grzeskowiak and J. F. Winkel, J. Electrochem. Soc., 154, J289 (2007).

9. A. R. Verma and P. Krishna, Polymorphism and Polytypism in Crystals, John Wiley \& Sons, New York (1966).

10. D. P. Varn, G. S. Canright, and J. P. Crutchfield, Acta Crystallogr., Sect. B: Struct. Sci., 63, 169 (2007).

11. M. T. Sebastian, J. Mater. Sci., 23, 2014 (1988)

12. M. T. Sebastian and P. Krishna, Phys. Status Solidi A, 79, 271 (1983).

13. M. T. Sebastian and P. Krishna, Solid State Commun., 48, 879 (1983).

14. M. T. Sebastian and P. Krishna, J. Cryst. Growth, 66, 586 (1984).

15. S. Koch, Acta Mineral. Petrogr. 11, 11 (1958).

16. E. Nitta, M. Kimata, M. Hoshino, T. Echigo, S. Hamasaki, N. Nishida, M. Shimizu, and T. Akasaka, J. Mineralogical and Petrographical Sciences, 103, 145 (2008).

17. C. Frondel and C. Palache, Science, 107, 602 (1948)

18. C. Frondel and C. Palache, Am. Mineral., 35, 29 (1950).

19. D. P. Varn, G. S. Canright, and J. P. Crutchfield, Phys. Rev. B, 66, 174110 (2002)

20. D. P. Varn and G. S. Canright, Acta Crystallogr., Sect. A: Found. Crystallogr., 57, 
4 (2001).

21. I. T. Steinberger, I. Kiflawi, Z. H. Kalman, and S. Mardix, Philos. Mag., 27, 159 (1973).

22. M. E. Fleet, Am. Mineral., 62, 540 (1977),

23. V. B. Reddy and K. T. Reilly, U.S. Pat. 5,711,898 (1998)

24. K. D. Budd, U.S. Pat. 5,418,062 (1995); K. T. Reilly, R. G. W. Gingerich, and C. S. Poss, U.S. Pat. 5,009,808 (1991): R. E. Karam and A. Wold, U.S. Pat. 5,309,071 (1994); Y. Kamei, Y. Kamiyama, M. Mafume, and Y. Katsuki, U.S. Pat. 5,841,738 (1998); V. B. Reddy and K. T. Reilly, U.S. Pat. 5,702,643 (1997); N. E. Brese and K. T. Reilly, U.S. Pat. 5,643,496 (1997).

25. H. Takemura, Y. Shimizu, T. Takahara, and M. Oikawa, U.S. Pat. 6,248,261 (2001). 26. http://www.ccdc.cam.ac.uk/, last accessed 2009.
27. M. T. Sebastian and P. Krishna, Pramana, 23, 395 (1984).

28. M. T. Sebastian and P. Krishna, Random, Non-Random and Periodic Faulting in Crystals, Gordon and Breach Science, Amsterdam (1994).

29. G. Shachar, S. Mardix, and I. T. Steinberger, J. Appl. Phys., 39, 2485 (1968).

30. Z. Shuyuan, G. Changxin, L. Bilin, and T. Shun, Philos. Mag. A, 62, 387 (1990)

31. M. Akizuki, Am. Mineral., 66, 1006 (1981).

32. V. Šrot, A. Rečnik, C. Scheu, S. Šturm, and B. Mirtič, Am. Mineral., 88, 1809 (2003).

33. S. I. Ben-Abraham, J. Appl. Phys., 36, 2096 (1965)

34. O. Brafman, E. Alexander, B. S. Fraenkel, Z. H. Kalman, and I. T. Steinberger, J. Appl. Phys., 35, 1855 (1964)

35. P. Goldberg, J. Appl. Phys., 32, 1520 (1961). 